\section{Definition of a standard protocol to determine the growth potential of Listeria monocytogenes and Yersinia enterocolitica in pork sausage produced in Abruzzo Region, Italy}

\author{
Anna Franca Sperandii, \\ Diana Neri, Romina Romantini, \\ Gino Angelo Santarelli, \\ Vincenza Prencipe \\ Institute for Experimental Veterinary \\ Medicine of Abruzzo and Molise, Teramo, \\ Italy
}

\section{Abstract}

Pork meat products consumed raw or after a short period of fermentation can be considered at risk for food safety. Sausages (fresh sausage made from pork meat) are produced in several Italian regions, with variation in ingredients. In some Italian Regions, including Abruzzo, these products are frequently consumed raw or undercooked, after a variable period of fermentation. The European Community food regulation promotes the use of challenge tests to determine safety levels. This study is aimed to ensure safety of Abruzzo's sausages, compared with growth potential ( $\delta$ ) of Listeria monocytogenes and Yersinia enterocolitica, and also aims to define an experimental standard protocol document to carry out challenge tests. Guidelines classify ready-to-eat foods in categories that are able to support $\left(\delta>0.5 \log _{10}\right.$ $\mathrm{ufc} / \mathrm{g})$ and not support $\left(\delta \leq 0.5 \log _{10} \mathrm{ufc} / \mathrm{g}\right)$ the growth of Listeria monocytogenes. The products were manufactured according to traditional recipes and were contaminated in laboratory. Results from the experiment yielded information useful to assess the ability of these products to support the growth of pathogenic microorganisms. The batches of sausages were stored at $8,12,18$ and $20^{\circ} \mathrm{C}$ to get statistical evaluation. The results showed that, despite the conditioning of the storage temperature and the level of water activity, both organisms remain in the product in concentrations similar to those leading or being able to increase its charge. In particular, the period of greatest consumption of this product (7/8 days of preparation) corresponds to the period of greatest growth of pathogenic microorganisms studied, except for those stored at a temperature of $8^{\circ} \mathrm{C}$, which are safer for the consumer.

\section{Introduzione}

La salsiccia fresca è un insaccato di carne cruda macinata, definita come preparato a base di carne, ossia carni fresche, incluse le carni ridotte in frammenti, che hanno subito un'aggiunta di prodotti o trattamenti non sufficienti a modificare la struttura muscolo-fibrosa interna della carne e a eliminare quindi le caratteristiche delle carni fresche (Commissione Europea, 2004). I prodotti a base di carne suina consumati crudi, poco cotti o poco stagionati sono considerati alimenti a rischio di Listeria monocytogenes. Anche Yersinia enterocolitica è un agente patogeno frequentemente riscontrato nelle carni suine (EFSA, 2010).

Il settore agro alimentare italiano è caratterizzato fortemente da produzioni alimentari tipiche preparate in modo tradizionale. La carenza di dati scientificamente documentati sul grado di sicurezza di tali prodotti, rispetto ad agenti microbici, causa di infezioni alimentari, penalizza questo tipo di produzione in quanto non consente di valutarne e gestirne il grado di rischio (Aquilanti et al., 2007; Cibotti et al., 2008; Silvestri et al., 2007).

La temperatura viene identificata come uno dei fattori che influenza la crescita di $L$. monocytogenes nei prodotti alimentari pronti per il consumo [ready-to-eat (RTE)] durante la durata di conservazione (shelf-life) (EFSA, 2010). Il monitoraggio delle temperature durante le fasi di produzione e vendita al dettaglio del prodotto è di solito documentato, ma non può essere riscontrato durante la distribuzione e la successiva conservazione domestica. La normativa alimentare dell'Unione Europea (Commissione Europea, 2005) prevede l'impiego di challenge test per determinare i livelli di sicurezza dei prodotti pronti al consumo, che non devono superare i $100 \mathrm{ufc/g}$ al termine della vita commerciale.

La conduzione del challenge test effettuato sul prodotto ha permesso di descrivere, tramite il calcolo del potenziale di crescita ( $\delta$ ), la capacità del prodotto di contrastare in modo attivo, a diverse condizioni di conservazione, la presenza di Listeria monocytogenes e Yersinia enterocolitica al variare di fattori quali acqua libera $\left(\mathrm{a}_{\mathrm{w}}\right)$ e temperatura $\left(\mathrm{T}^{\circ}\right)$. Lo studio ha previsto la produzione della salsiccia di carne suina secondo le modalità della tradizione abruzzese e l'esecuzione del challenge test preceduto da una fase di progettazione, nel corso della quale sono state elaborate una serie di considerazioni relative allo specifico alimento. La sperimentazione è stata condotta in diversi giorni e per ogni lotto contaminato di prodotto è stato effettuato il corrispettivo controllo. Tutte le produzioni sono state stoccate a diverse temperature $\left(8,12,18\right.$ e $\left.20^{\circ} \mathrm{C}\right)$ scelte in funzione delle condizioni in cui il prodotto è conservato nel normale utilizzo fino al momento
Correspondence: Anna Franca Sperandii, Institute for Experimental Veterinary Medicine of Abruzzo and Molise G. Caporale, via Campo Boario, 64100 Teramo, Italy.

Tel. +39.0861.332467.

E-mail: a.sperandii@izs.it

Key words: Challenge test; Listeria monocytogenes; Yersinia enterocolitica; Growth potential $(\delta)$; Pork sausage.

Note: In memory of colleague Vincenza Prencipe (10/01/2014): "A very rare privilege to work with you. For your smile, availability and enthusiasm. Thanks."

Received for publication: 16 July 2014 .

Revision received: 2 July 2015.

Accepted for publication: 8 July 2015

This work is licensed under a Creative Commons Attribution-NonCommercial 3.0 International License (CC BY-NC 3.0).

del consumo (temperature di refrigerazione alla vendita, domestica e temperature di abuso). La varietà di considerate è stata definita anche in relazione alla necessità di ottenere informazioni dettagliate sulla cinetica dei microrganismi, finalizzate allo sviluppo di modelli di microbiologia predittiva.

L'obiettivo del lavoro è di presentare un protocollo sperimentale standard per la conduzione di challenge test, al fine di ottenere informazioni necessarie e riproducibili per la valutazione del rischio dell'intero processo di produzione di insaccati freschi. Tale protocollo è stato redatto in seguito allo studio del processo produttivo, delle caratteristiche del prodotto, del confezionamento e dello stoccaggio. Le fasi del challenge test, dalla scelta dei ceppi alla contaminazione della matrice alimentare, sono state definite in accordo con la Guida tecnica del Laboratorio di riferimento europeo per Listeria monocytogenes (ANSES, 2008).

Le sperimentazioni sono state eseguite presso il Laboratorio di Trasformazioni Sperimentali, struttura ad alto contenimento biologico, dell'Istituto Zooprofilattico Sperimentale dell'Abruzzo e del Molise (IZSAM) G. Caporale (Teramo).

\section{Materiali e Metodi}

Al fine di riprodurre il processo produttivo della salsiccia, sono stati effettuati sopralluoghi presso laboratori di produzione locali per studiare le variabili di prodotto e di processo.

\section{Rilievo delle caratteristiche del prodotto}

La salsiccia di suino della tradizione abruz- 
zese è costituita generalmente da carni fresche di suino e da grasso tritate con l'aggiunta di aromi e spezie. Le salsicce dalla pezzatura di circa 70 grammi si presentano in filze (Figura 1). Solitamente di consistenza morbida, $\left(a_{w}\right.$ 0,967 ) vengono consumate sia crude che poco cotte 0 dopo un breve periodo di conservazione con una shelf-life che può variare da 7 a 15 giorni (7 giorni: periodo di maggior consumo). La produzione eseguita in laboratori annessi a macellerie è molto diffusa e in ogni caso il prodotto è destinato a essere conservato nella fase di vendita a temperatura controllata.

\section{Rilievo del processo produttivo}

La sperimentazione ha previsto la realizzazione di salsicce secondo il processo produttivo tradizionale. Sono state utilizzate carni fresche di suino, per l'80\% ottenute da ritagli e rifilature (spalla, collo, pancetta e coscia) e grasso per il restante 20\% (lardello di schiena e pancetta).

Le fasi di produzione hanno previsto: mondatura, pesata e macinazione di carne e grasso, preparazione della concia a base di sale (22 $\mathrm{g} / \mathrm{kg}$ ), pepe macinato ( $\mathrm{g} / \mathrm{kg}$ ) e buccia di arancia disidratata, miscelazione, insacco in budello naturale, legatura e foratura.

Dalle salsicce, prodotte in diversi giorni, sono stati preparati lotti di campioni contaminati artificialmente con differenti livelli di concentrazioni di $L$. monocytogenes e $Y$. enterocolitica, e i rispettivi lotti di campioni di controllo non contaminati. I lotti sono stati stoccati in celle dedicate a condizioni diverse di temperatura: $8( \pm 1)^{\circ} \mathrm{C}, 12( \pm 1)^{\circ} \mathrm{C}, 18( \pm 1)^{\circ} \mathrm{C}$ e 20 $( \pm 1)^{\circ} \mathrm{C}$ (Tabella 1$)$.

\section{Durata dello studio e campiona- mento}

Lo studio ha previsto un periodo di conservazione del prodotto, commercialmente non superiore a 14-15 giorni, della durata di 20 giorni, prolungando di $1 / 4$ la shelf-life. Il campionamento ha previsto prelievi eseguiti fino al $20^{\circ}$ giorno sia per i campioni contaminati sia di controllo. Per il primo e secondo lotto durante i primi 7 giorni di sperimentazione i prelievi sono stati eseguiti giornalmente per essere condotti in seguito nei giorni 9, 12, 14, 17 e 20 , per un totale di 12 giorni di campionamento.

Per le successive produzioni di salsicce del terzo e del quarto lotto, il campionamento è stato incrementato di un prelievo al giorno per i primi 5 giorni, per un totale di 17 giorni di campionamento. L'incremento si è reso necessario al fine di ottenere maggiori informazioni sulle dinamiche di crescita dei microrganismi in questione, particolarmente nella fase di crescita esponenziale e a diverse temperature. L'ottenimento di questi dati è stato utilizzato per lo sviluppo di specifici modelli di microbiologia predittiva. Per ogni campionamento sono state prelevate tre salsicce da ogni lotto di prodotto in esame.

\section{Preparazione dell'inoculo}

Per la preparazione dell'inoculo di ciascun patogeno, il protocollo sperimentale ha fatto riferimento alla Procedura operativa standard IZSTE B 3.1.2 Bozza SOP124 Challenge test microbiologico - Ceppi batterici: criteri di selezione, curve di crescita e adattamento per la preparazione di sospensioni di inoculo per unità di prova artificialmente contaminate redatta in accordo con le linee guida del Laboratorio Europeo di Riferimento.

I ceppi utilizzati sono stati: L. monocytogenes [ceppo di riferimento ATCC 7644 e due ceppi appartenenti ai sierotipi 1/2A e 1/2C isolati da matrice analoga al prodotto oggetto dello studio]; $Y$. enterocolitica [ceppo di riferimento, appartenente a una collezione internazionale, NCTC 10463 e due ceppi di campo (IZS 20012 e IZS1735) isolati da tonsille di suino].

I ceppi conservati a $-80^{\circ} \mathrm{C}$ sono stati singolarmente rivitalizzati in Brain heart infusion (BHI) in aerobiosi e seminati su agar sangue. Successivamente, per ogni ceppo sono state preparate brodocolture in $\mathrm{BHI}$ incubate a $37^{\circ} \mathrm{C}$ per $24 \mathrm{~h}$. Dalle brodocolture ottenute sono state allestite nuove subcolture alle condizioni di adattamento in BHI modificato con $\mathrm{pH} 7,4 \mathrm{e}$ $\mathrm{NaCl} 2,3 \%$ (caratteristiche del prodotto) e alle temperature di $8-12^{\circ} \mathrm{C}$ (temperature di normale conservazione) e $20-18^{\circ} \mathrm{C}$ (temperature di abuso). Al raggiungimento della fase stazionaria, per entrambe le specie batteriche e per le temperature previste, sono state ottenute sospensioni di inoculo costituite dall'unione di parti uguali delle brodocolture adattate dei tre ceppi selezionati. Le sospensioni sono state successivamente diluite per ottenere concentrazioni d'uso utili per l'inoculo del prodotto e verificate con la titolazione del ceppo (conta in piastra). Le prove sperimentali hanno previsto la contaminazione del prodotto con concentrazioni variabili da $1 \times 10^{4}$ ufc/g (lotti 1-2) a $1 \times 10^{2}$ ufc/g (lotti 3-4) (Tabella 1). I livelli di contaminazione hanno rappresentato la concentrazione massima e la più frequente rilevate in questi prodotti (Barbieri e Bonardi, 2007).

Al fine di ridurre le modifiche delle proprietà chimico-fisiche del prodotto, l'inoculo inferiore all'1\% del volume delle unità di prova contaminate è stato effettuato mediante siringa, distribuendo in profondità $0.4 \mathrm{~mL}$ di ciascuna sospensione batterica, in tre punti distinti di ogni singola salsiccia. I campioni di controllo sono stati inoculati con il medesimo quantitativo di soluzione fisiologica sterile.

\section{Determinazioni analitiche}

La contaminazione naturale dei due patogeni è stata valutata sui campioni di controllo al T0 mediante ricerca di Yersinia enterocolitica ISO 10273:2003 e di L. monocytogenes ISO 11290-1:96/Amendment 1:2004 (ISO, 2003, 2004c). Sui campioni di controllo, inoltre, per tutti i giorni di campionamento sono stati determinati i seguenti parametri: numerazione lattobacilli ISO 15214:1998, numerazione micrococchi ISO 6888-1:1999 (ISO, 1998, 1999), numerazione enterococchi NO 68 4th Ed. 2004 - Nordic Committee - on Food Analisys, determinazione $\mathrm{a}_{\mathrm{w}}$ ISO 21807:2004 (ISO, 2004a), determinazione $\mathrm{pH}$ metodo interno.

Per la determinazione dell'acqua libera $a_{w}$ è stato utilizzato AquaLab 4TE (Decagon Devices, Pullman, WA, USA), per il pH, il pHmetro Seven easy [Mettler Toledo, Novate Milanese (MI), Italia].

Nei campioni contaminati la valutazione dell'andamento è stata effettuata con numerazione L. monocytogenes ISO 112902:98/Amendment 1:2004 (ISO, 2004b) e numerazione Yersinia spp. metodo interno. Per tutte le determinazioni previste, l'intera aliquota di salsiccia è stata omogeneizzata utilizzando il Knife Mill Grindomix GM200 (Retsch, Haan, Germania), prelevandone successivamente i quantitativi necessari. Ogni determinazione è stata eseguita in triplicato e il risultato è stato calcolato come valore medio delle tre misurazioni.

Tabella 1. Lotti di stoccaggio alle diverse temperature e concentrazioni di Listeria monocytogenes e Yersinia enterocolitica.

\begin{tabular}{lccc} 
Lotti & $\begin{array}{c}\text { Temperature di stoccaggio }\left({ }^{\circ} \mathrm{C}\right) \\
\text { Livelli di contaminavione }\end{array}$ & $\begin{array}{c}\text { Livelli di contaminavione } \\
\text { di } Y . \text { enterocolitica (ufc/g) }\end{array}$ \\
\hline $1-2$ & $8 / 20$ & $1 \times 10^{4}$ & $1 \times 10^{4}$ \\
$3-4$ & $12 / 18$ & $1 \times 10^{2}$ & $1 \times 10^{2}$ \\
\hline Controlli $1-2$ & $8 / 20$ & - & - \\
Controlli 3-4 & $12 / 18$ & - & - \\
\hline
\end{tabular}

L. monocytogenes, Listeria monocytogenes; Y. enterocolitica, Yersinia enterocolitica. 


\section{Calcolo del potenziale di crescita}

Il potenziale di crescita $\delta$ è stato calcolato per tutte le temperature, sia per $L$. monocytogenes che per $Y$. Enterocolitica, come differenza Nt-N0, dove Nt è la concentrazione batterica espressa in $\log _{10} \mathrm{ufc} / \mathrm{g}$ al tempo $7 \mathrm{gg}$ (periodo di maggior consumo) e 20 gg (fine sperimentazione) e N0 è la concentrazione batterica al tempo 0 (inoculo).

\section{Risultati}

Attraverso la raccolta e l'interpretazione dei parametri microbiologici, chimico-fisici e merceologici sono state ottenute informazioni utili per standardizzare e migliorare i processi produttivi.

Nelle Figure 2 e 3, relative alla dinamica di comportamento di L. monocytogenes e $Y$. enterocolitica alle temperature considerate, vengono rappresentati i valori di $\mathrm{a}_{\mathrm{w}}$ con uno smoo- thing dei dati ottenuti, effettuato attraverso una retta di regressione lineare (dove $a_{w}$ è funzione lineare del tempo). In entrambi i lotti contaminati, sia $L$. monocytogenes che $Y$. enterocolitica hanno mostrato un incremento della carica maggiore nelle salsicce conservate alle temperature ambientali. I massimi livelli di crescita sono stati raggiunti da $L$. monocytogenes in 12 giorni a $20^{\circ} \mathrm{C}\left(+2,46 \log _{10} \mathrm{ufc} / \mathrm{g}\right.$ rispetto ai livelli di contaminazione iniziale di $\left.10^{4} \mathrm{ufc} / \mathrm{g}\right)$ e in 4 giorni a $18^{\circ} \mathrm{C}\left(+2,91 \log _{10} \mathrm{ufc} / \mathrm{g}\right.$ rispetto a $\left.10^{2} \mathrm{ufc} / \mathrm{g}\right)$. $Y$. enterocolitica ha raggiunto i massimi livelli di contaminazione a 7 giorni nelle salsicce conservate a $20^{\circ} \mathrm{C}(+1,63$ $\log _{10}$ ufc/g) e a 5 giorni nelle salsicce conservate a $18^{\circ} \mathrm{C}\left(+3,58 \log _{10} \mathrm{ufc} / \mathrm{g}\right)$. Nei prodotti conservati a temperature di refrigerazione l'andamento ha mostrato uno sviluppo dei microrganismi inoculati più contenuto, in particolare le salsicce conservate a $12^{\circ} \mathrm{C}$ hanno raggiunto il livello massimo di $L$. monocytogenes in 14 giorni $\left(+2,46 \log _{10}\right.$ ufc/g rispetto ai livelli di contaminazione iniziale di $10^{2} \mathrm{ufc} / \mathrm{g}$ ) e di $Y$. entero- colitica in 5 giorni $\left(+1,99 \log _{10} \mathrm{ufc} / \mathrm{g}\right.$ rispetto alla contaminazione iniziale $10^{2} \mathrm{ufc} / \mathrm{g}$ ). Nei prodotti conservati a $8^{\circ} \mathrm{C}$ si è osservato uno scarso incremento di crescita nelle salsicce contaminate con $Y$. enterocolitica, il massimo livello di contaminazione è stato raggiunto il giorno 9 con aumento dei livelli di crescita di $0,63 \log _{10} \mathrm{ufc} / \mathrm{g}$. Nelle salsicce contaminate con L. monocytogenes, nelle prime $24 \mathrm{~h}$ dopo l'inoculo, si è verificata una inattesa riduzione della concentrazione di circa $1 \log _{10}$ ufc/g (lento adattamento del patogeno nel prodotto). Il valore massimo è stato raggiunto il giorno 17, risultando simile al livello di contaminazione iniziale di $10^{4} \mathrm{ufc} / \mathrm{g}$. Pertanto, per il calcolo del potenziale di crescita a $8^{\circ} \mathrm{C}$ si è preferito considerare quale N0 la concentrazione rilevata al giorno $1\left(3,28 \log _{10} \mathrm{ufc} / \mathrm{g}\right)$ piuttosto che quella misurata al giorno 0 . Il potenziale di crescita nei prodotti contaminati sia con L. monocytogenes che con $Y$. enterocolitica è risultato $<0,5 \log _{10}$ ufc/g nei campioni conservati a $8^{\circ} \mathrm{C}$ sia a 7 che a 20 giorni (Tabella 2). In Figura 4

Tabella 2. Calcolo del potenziale di crescita $\delta$ per tutte le temperature, a 7 e 20 giorni.

\begin{tabular}{|c|c|c|c|c|}
\hline Lotti & Giorni & $\mathrm{T} \pm 1\left({ }^{\circ} \mathrm{C}\right)$ & $\begin{array}{c}\text { Potenviale di crescita di } \\
\text { L. monocytogenes }\end{array}$ & $\begin{array}{c}\text { Potenziale di crescita di } \\
\text { Y. enterocolitica }\end{array}$ \\
\hline 1 & 7 & 8 & $-0,002$ & $-0,064$ \\
\hline 1 & 20 & 8 & $2 x+8$ & 0,423 \\
\hline 2 & 7 & 20 & 1,460 & 1,630 \\
\hline 2 & 20 & 20 & 0,585 & $-0,755$ \\
\hline 3 & 7 & 12 & 1,300 & 1,103 \\
\hline 3 & 20 & 12 & 0,522 & 1,240 \\
\hline 4 & 7 & 18 & 2,324 & 3,206 \\
\hline 4 & 20 & 18 & 1,609 & 2,268 \\
\hline
\end{tabular}

T, temperatura; L. monocytogenes, Listeria monocytogenes; Y. enterocolitica, Yersinia enterocolitica.

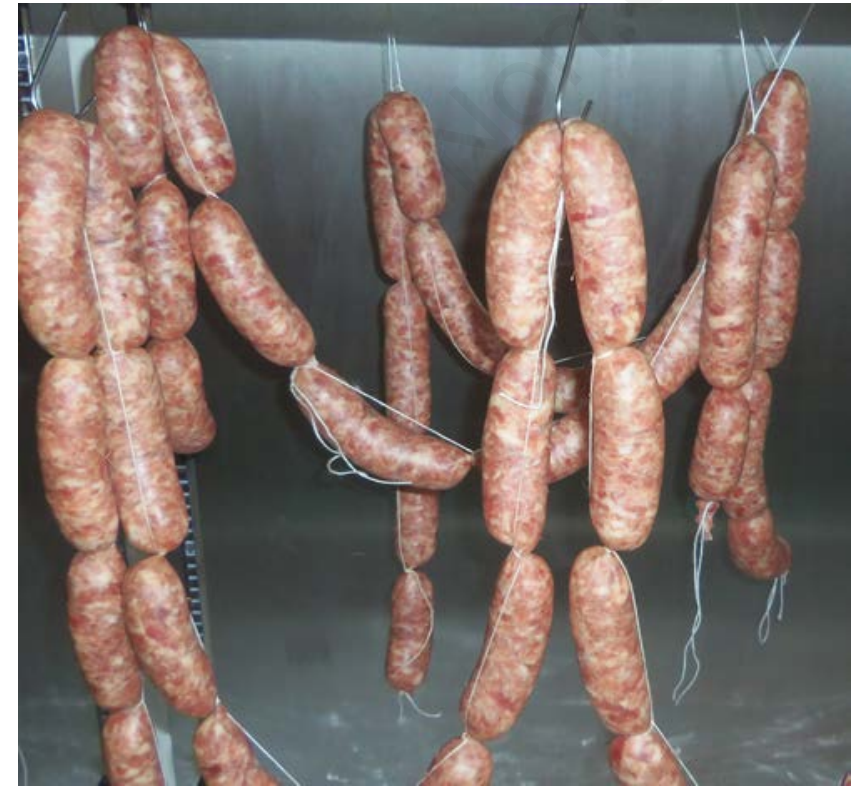

Figura 1. Filze di salsicce.

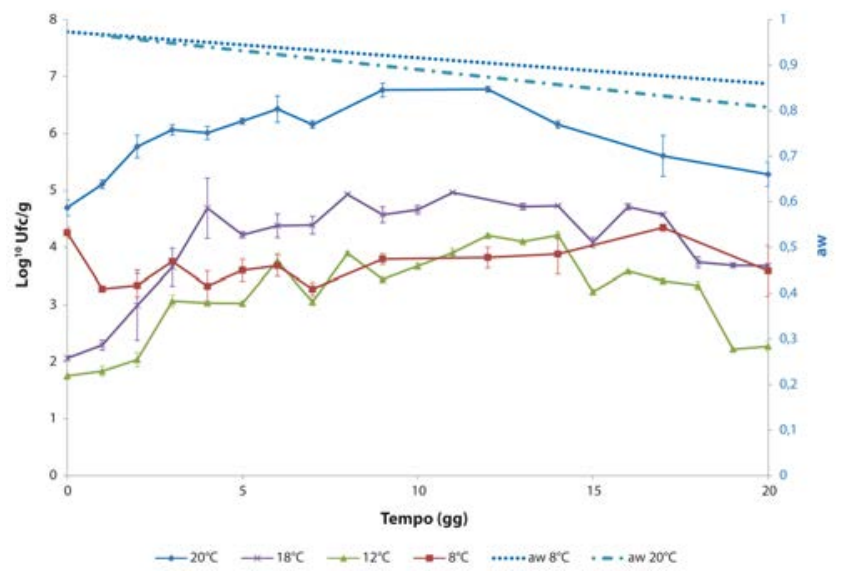

Figura 2. Valori di acqua libera per Listeria monocytogenes. 
è rappresentato l'andamento della flora microbica autoctona rilevata considerando le temperature di $8^{\circ} \mathrm{C}$ (minima) e $20^{\circ} \mathrm{C}$ (massima). In Tabella 3 è riportato l'andamento del $\mathrm{pH}$ rilevato nei campioni conservati alle diverse temperature $8,20,12,18^{\circ} \mathrm{C}$, risultando poco variabile in tutti i campioni, valore medio di 5,96.

\section{Discussione}

La conduzione della sperimentazione con diverse temperature di stoccaggio e diversi livelli di contaminazione iniziale ha permesso di valutare il comportamento dei patogeni inoculati nel prodotto simulando condizioni ambientali e di contaminazione variabili.

Dall'analisi si evidenzia come la temperatu- ra svolga un ruolo determinante nel contenimento della carica dei patogeni in esame in associazione allo sviluppo della flora microbica autoctona, in particolare dei lattobacilli (Vignolo et al., 1993). Inoltre, anche la composizione del prodotto (parte lipidica) oltre a condizionare gli aspetti organolettici, potrebbe avere una certa influenza sul loro decremento (Mbandi et al., 2004).

È stata rilevata una graduale e costante riduzione dell' $\mathrm{a}_{\mathrm{w}}$ in tutti i campioni a tutte le temperature valutate e, come prevedibile, alle temperature maggiori il decremento dei valori di $a_{w}$ è stato più rapido (sottrazione di acqua libera dovuta anche alla presenza di sale).

Il $\mathrm{pH}$, risultato invariato nel tempo, attesta la natura d'insaccato fresco del prodotto in esame (Orefice, 2014). Si è potuto osservare una diminuzione dello sviluppo dei batteri inoculati anche prima che l'a $\mathrm{a}_{\mathrm{w}}$ raggiunga valori
$<0,90$, pertanto, in assenza di livelli di pH e $\mathrm{a}_{\mathrm{w}}$ in grado di determinare l'inattivazione, la competizione con la flora microbica autoctona (lattica) è la più probabile concausa di decremento della crescita di $L$. monocytogenes e $Y$. enterocolitica.

Sulla base della classificazione degli alimenti pronti per il consumo (ANSES, 2008), è possibile affermare che il prodotto esaminato, pur non presentando caratteristiche intrinseche né processi tecnologici di produzione tali da abbattere la concentrazione dei patogeni studiati, sia a 7 che a 20 giorni dalla produzione e a temperature di stoccaggio di $8^{\circ} \mathrm{C}$, risulta non essere favorevole $\left(\delta \leq 0,5 \log _{10} \mathrm{ufc} / \mathrm{g}\right)$ alla crescita di L. monocytogenes. Applicando gli stessi parametri a Yersinia enterocolitica, per la quale però non esistono linee guida specifiche, è possibile giungere alle stesse valutazioni.

Tabella 3. Andamento del pH nei campioni conservati alle diverse temperature.

\begin{tabular}{|c|c|c|c|c|}
\hline Giorni & $\mathrm{pH} \pm \mathrm{DS} 8^{\circ} \mathrm{C}$ & $\mathrm{pH} \pm \mathrm{DS} 12^{\circ} \mathrm{C}$ & $\mathrm{pH} \pm \mathrm{DS} 18^{\circ} \mathrm{C}$ & $\mathrm{pH} \pm \mathrm{DS} 20^{\circ} \mathrm{C}$ \\
\hline 0 & $6,00 \pm 0,07$ & $5,92 \pm 0,03$ & $5,92 \pm 0,04$ & $6,00 \pm 0,02$ \\
\hline 1 & $5,72 \pm 0,06$ & $6,28 \pm 0,06$ & $6,28 \pm 0,05$ & $5,64 \pm 0,02$ \\
\hline 2 & $5,98 \pm 0,01$ & $5,94 \pm 0,01$ & $5,92 \pm 0,01$ & $5,93 \pm 0,01$ \\
\hline 3 & $5,97 \pm 0,02$ & $5,9 \pm 0,03$ & $5,88 \pm 0,04$ & $5,80 \pm 0,10$ \\
\hline 4 & $5,83 \pm 0,02$ & $5,88 \pm 0,02$ & $5,85 \pm 0,04$ & $5,93 \pm 0,05$ \\
\hline 5 & $6,00 \pm 0,06$ & $5,84 \pm 0,04$ & $5,82 \pm 0,06$ & $5,83 \pm 0,05$ \\
\hline 6 & $5,96 \pm 0,08$ & $5,71 \pm 0,09$ & $5,77 \pm 0,05$ & $5,69 \pm 0,10$ \\
\hline 7 & $6,01 \pm 0,04$ & $5,67 \pm 0,02$ & $5,69 \pm 0,04$ & $5,63 \pm 0,12$ \\
\hline 9 & $6,34 \pm 0,08$ & $6,11 \pm 0,07$ & $6,15 \pm 0,05$ & $6,10 \pm 0,09$ \\
\hline 12 & $6,08 \pm 0,07$ & $5,87 \pm 0,08$ & $5,84 \pm 0,07$ & $5,89 \pm 0,04$ \\
\hline 14 & $6,14 \pm 0,08$ & $6,39 \pm 0,09$ & $6,33 \pm 0,05$ & $6,09 \pm 0,12$ \\
\hline 17 & $5,95 \pm 0,08$ & $5,70 \pm 0,09$ & $5,71 \pm 0,08$ & $5,71 \pm 0,08$ \\
\hline 20 & $6,09 \pm 0,07$ & $5,70 \pm 0,04$ & $5,62 \pm 0,11$ & $5,86 \pm 0,06$ \\
\hline
\end{tabular}

DS, deviazione standard.

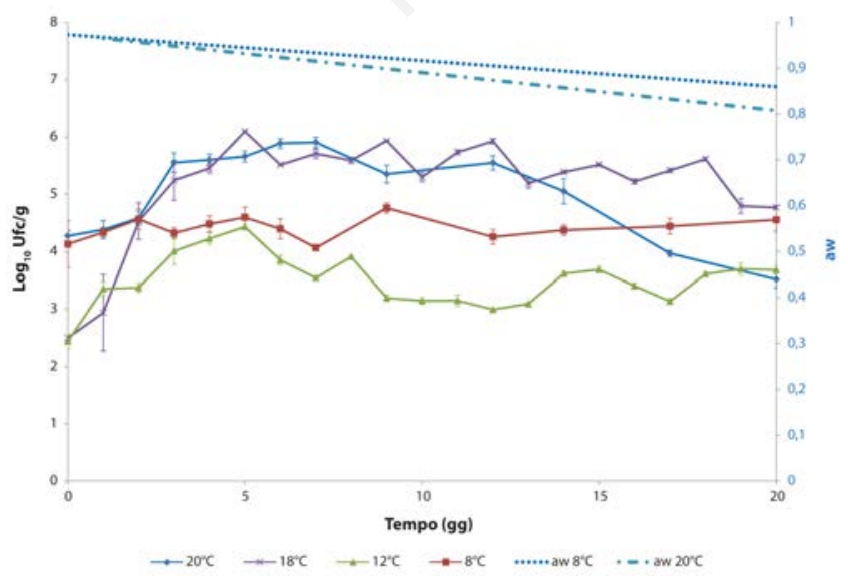

Figura 3. Valori di acqua libera per Yersinia enterocolitica.

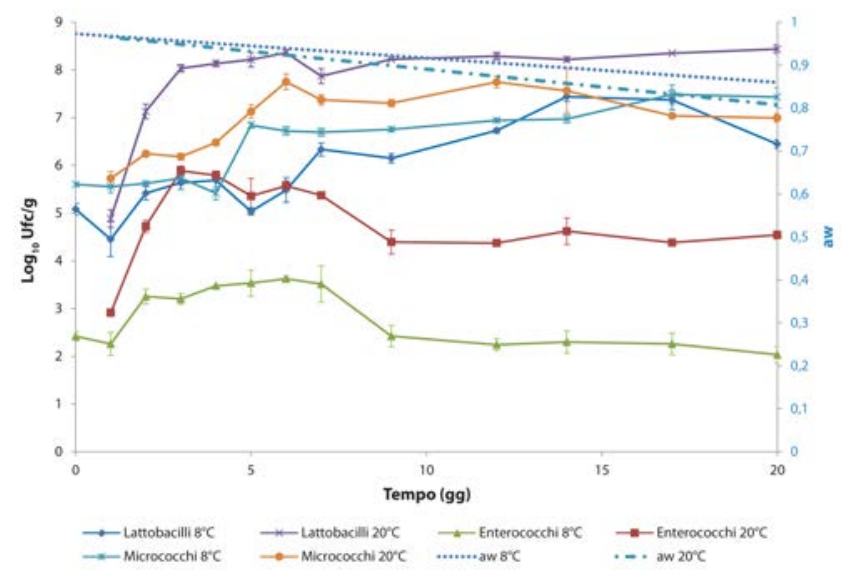

Figura 4. Andamento della flora microbica autoctona rilevata considerando le temperature di $8^{\circ} \mathrm{C}$ (minima) e $20^{\circ} \mathrm{C}$ (massima). 
mi during its natural fermentation. Int $\mathrm{J}$ Food Microbiol 120:136-45.

Barbieri S, Bonardi S, 2007. Il ruolo del suino nell'epidemiologia della tossinfezione alimentare da Yersinia enterocolitica. Ann Fac Med Vet Parma 27:43-60.

tale per challenge test, specifico per un prodotto tradizionale come la salsiccia abruzzese, ha permesso di ottenere risultati utilizzabili per la definizione di un'adeguata shelf-life e generare dati che saranno impiegati per la produzione di modelli matematici predittivi. In particolare, è stato possibile stabilire che, ai fini della sicurezza del consumatore, è auspicabile che il prodotto salsiccia fresca di suino della tradizione abruzzese sia preparato secondo quanto indicato dalla ricetta tipica (percentuale di sale e aromi per favorire l'azione antibatterica) e conservato a temperature non superiori a $8^{\circ} \mathrm{C}$, inclusa la fase di vendita, per un periodo di 20 giorni dalla data di produzione. Spesso l'insufficienza di dati scientifici disponibili validati non permette di evidenziare i reali livelli di sicurezza dei prodotti tradizionali. La progettazione e la valutazione di studi sui challenge test è un compito complesso che dipende da vari fattori che se sottovalutati potrebbero portare a produrre dati e conclusioni non reali. Il presente studio fornisce informazioni pratiche per standardizzare le fasi cruciali di challenge test per ottenere risultati ripetibili e confrontabili.

\section{Bibliografia}

ANSES, 2008. Technical guidance document on shelf-life studies for Listeria monocytogenes in ready-to-eat foods. Agence nationale de sécurité sanitaire de l'alimentation, de l'environnement et du travail ed., Maisons-Alfort Cedex, France.

Aquilanti L, Santarelli S, Silvestri G, Osimani A, Petruzzelli A, Clementi F, 2007. The microbial ecology of a typical Italian sala-
Organizzazione Internazionale per la Standardizzazione ed., Ginevra, Svizzera.

ISO, 2003. Microbiology of food and animal feeding stuff: microbiology of food and animal feeding stuffs. Horizontal method for the detection of presumptive pathogenic Yersinia enterocolitica. Norma ISO 10273. Organizzazione Internazionale per la Standardizzazione ed., Ginevra, Svizzera.

ISO, 2004a. Microbiology of food and animal feeding stuff- Determination of water activity. Norma ISO 21807. Organizzazione Internazionale per la Standardizzazione ed., Ginevra, Svizzera.

ISO, 2004b. Modification of the enumeration medium. Norma ISO 11290-2:1998/Amd 1:2004. Organizzazione Internazionale per la Standardizzazione ed., Ginevra, Svizzera.

ISO, 2004c. Modification of the isolation media and the haemolysis test, and inclusion of precision data. Norma ISO 112901:1996/Amd 1:2004. Organizzazione Internazionale per la Standardizzazione ed., Ginevra, Svizzera.

Mbandi E, Brywig M, Shelef L, 2004. Antilisterial effects of free fatty acids and monolaurin in beef emulsions and hot dogs. Food Microbiol 21:815-8.

Orefice L, 2014. L'igiene della produzione e della conservazione degli alimenti con particolare riguardo al settore delle carni. Istituto Superiore di Sanità, Roma, Italia.

Silvestri G, Santarelli S, Aquilanti L, 2007. Investigation of the ecology of ciauscolo, a traditional Italian salami, by culturedependent techniques and PCR-DGGE. Meat Sci 77:413-23.

Vignolo GM, Suriani F, Pesce de Ruiz Holgado A, Oliver G, 1993. Antibacterial activity of Lactobacillus strains isolated from dry fermented sausages. J Appl Bacteriol 75:3449. 